\title{
A Block-Cipher Mode of Operation for Parallelizable Message Authentication
}

\author{
John Black ${ }^{1}$ and Phillip Rogaway ${ }^{2}$ \\ 1 Dept. of Computer Science, University of Nevada, Reno NV 89557, USA, \\ jrb@cs.unr.edu, www.cs.unr.edu ${ }^{\sim}$ jrb \\ 2 Dept. of Computer Science, University of California, Davis, CA 95616, USA, \\ rogaway@cs.ucdavis.edu, www.cs.ucdavis.edu/ ${ }^{\sim}$ rogaway
}

\begin{abstract}
We define and analyze a simple and fully parallelizable blockcipher mode of operation for message authentication. Parallelizability does not come at the expense of serial efficiency: in a conventional, serial environment, the algorithm's speed is within a few percent of the (inherently sequential) CBC MAC. The new mode, PMAC, is deterministic, resembles a standard mode of operation (and not a CarterWegman MAC), works for strings of any bit length, employs a single block-cipher key, and uses just $\max \{1,\lceil|M| / n\rceil\}$ block-cipher calls to MAC a string $M \in\{0,1\}^{*}$ using an $n$-bit block cipher. We prove PMAC secure, quantifying an adversary's forgery probability in terms of the quality of the block cipher as a pseudorandom permutation.
\end{abstract}

\section{Introduction}

BACKGROUnd. Many popular message authentication codes (MACs), like the CBC MAC [17] and HMAC [1], are inherently sequential: one cannot process the $i$-th message block until all previous message blocks have been processed. This serial bottleneck becomes increasingly an issue as commodity processors offer up more and more parallelism, and as increases in network speeds outpace increases in the speed of cryptographic hardware. By now there would seem to be a significant interest in having a parallelizable MAC which performs well in both hardware and software, built from a block cipher like AES.

There are several approaches to the design of such an MAC. One is to generically construct a more parallelizable MAC from an arbitrary one. For example, one could begin with breaking the message $M[1] \cdots M[2 m]$ into $M^{\prime}=$ $M[1] M[3] \cdots M[2 m-1]$ and $M^{\prime \prime}=M[2] M[4] \cdots M[2 m]$ then separately MAC each half. But such an approach requires one to anticipate the maximal amount of parallelism one aims to extract. In the current work we are instead interested in fully parallelizable MACs: the amount of parallelism that can be extracted is effectively unbounded.

One idea for making a fully parallelizable MAC is to use the Carter-Wegman paradigm $[13,23]$, as in $[12,16,19]$, making sure to select a universal hashfunction family that is fully parallelizable. In fact, most universal hash functions that have been suggested are fully parallelizable. This approach is elegant 
and can lead to a nice MAC, but constructions for fast universal hash-functions have proven to be quite complex to specify or to implement well [7,9], and may be biased either towards hardware or towards software. Twenty years after the paradigm was introduced, we still do not know of a single Carter-Wegman MAC that actually gets used. So the current work goes back to giving a conventional mode, but one designed, this time around, for serial and parallel efficiency.

ThE XOR MAC. Bellare, Guérin and Rogaway introduced a parallelizable MAC in their XOR MACs [3]. The message $M$ is divided into pieces $M[1] \cdots M[\ell]$ of length less than the blocksize; for concreteness, think of each $M[i]$ as having 64 bits when the blocksize is $n=128$ bits. Each piece $M[i]$ is preceded by $[i]$, the number $i$ encoded as a 64-bit number, and to each $[i] \| M[i]$ one applies the block cipher $E$, keyed by the MAC key $K$. One more block is enciphered, it having a first bit of 1 and then a counter or random value in the remaining $n-1$ bits. The MAC is that counter or random value together with the $\mathrm{XOR}$ of all $\ell+1$ ciphertext blocks. Thus the MAC uses $\ell+1 \approx 2 m+1$ block-cipher invocations to authenticate a message of $m$ blocks of $n$ bits; one has paid for parallelizability at a cost of about a factor of two in serial speed. Further disadvantages include the need for randomness or state (conventional MACs are deterministic) and in the increased length of the MAC (because of the counter or random value that has to be included).

PMAC. Unlike the XOR MAC, our new algorithm, PMAC, doesn't waste any block-cipher invocations because of block-indices (nor for a counter or random values). Also, in the spirit of [11], we optimally deal with short final blocks; we correctly MAC messages of arbitrary and varying bit lengths. The result is that PMAC makes do with just $\lceil|M| / n\rceil$ block-cipher calls to MAC a nonempty message $M$ using an $n$-bit block cipher. PMAC is deterministic, freeing the user from having to provide a counter or random value, and making the MAC shorter. Overhead beyond the block-cipher calls has been aggressively optimized, so a serial implementation of PMAC runs just a few percent slower than the CBC MAC.

Besides the efficiency measures already mentioned, PMAC uses very little key-setup: one block-cipher call. (A few shifts and conditional xors are also used.) The PMAC key is a single key for the underlying blocks cipher; in particular, we forgo the need for key-separation techniques. Avoiding multiple block-cipher keys saves time because many block ciphers have significant key-setup costs.

Being so stingy with keys and block-cipher invocations takes significant care; note that even the traditional CBC MAC uses between one and four additional block-cipher calls, as well as additional key material, once it has been enriched to take care of messages of arbitrary lengths $[6,11,17,21]$. Of course avoiding this overhead doesn't matter much on long messages, but it is significant on short ones. And in many environments, short messages are common.

We prove PMAC secure, in the sense of reduction-based cryptography. Specifically, we prove that PMAC approximates a random function (and is therefore a good MAC) as long as the underlying block cipher approximates a random 
permutation. The actual results are quantitative; the security analysis is in the concrete-security paradigm.

PMAC was proposed in response to NIST's call for contributions for a first modes-of-operation workshop. Earlier versions of this writeup were submitted to NIST and posted to their website (Oct 2000, Apr 2001).

Additional Related Work. Building on [3], Gligor and Donescu describe a MAC they call the XECB MAC [14]. That MAC is not deterministic, it uses more block-cipher invocations, and it was not designed for messages of arbitrary bit length. But, like PMAC, it goes beyond the XOR MAC by combining a message index and a message block in a way other than encoding the two. In particular, [14] combines $i$ and $M[i]$ by adding to $M[i]$, modulo $2^{n}$, a secret multiple $i$. We combine $i$ and $M[i]$ by different means, to reduce overhead and obtain a better bound.

PMAC was also influenced by the variant of the XOR MAC due to Bernstein [8]. His algorithm is deterministic, and the way that the XOR MAC was made deterministic in [8] is similar to the way that PMAC has been made deterministic. Finally, there is also some similarity in appearance between PMAC and Jutla's IAPM encryption mode [18].

\section{Mathematical Preliminaries}

Notation. If $i \geq 1$ is an integer then $\mathrm{ntz}(i)$ is the number of trailing 0-bits in the binary representation of $i$. So, for example, $\operatorname{ntz}(7)=0$ and $\operatorname{ntz}(8)=3$. If $A \in\{0,1\}^{*}$ is a string then $|A|$ denotes its length in bits while $\|A\|_{n}=$ $\max \{1, \quad\lceil|A| / n\rceil\}$ denotes its length in $n$-bit blocks (where the empty string counts as one block). If $A=a_{n-1} \cdots a_{1} a_{0} \in\{0,1\}^{n}$ is a string (each $a_{i} \in\{0,1\}$ ) then $\operatorname{str} 2 \operatorname{num}(A)$ is the number $\sum_{i=0}^{n-1} 2^{i} a_{i}$. If $A, B \in\{0,1\}^{*}$ are equal-length strings than $A \oplus B$ is their bitwise xor. If $A \in\{0,1\}^{*}$ and $|A|<n$ then $\operatorname{pad}_{n}(A)$ is the string $A 10^{n-|A|-1}$. If $A \in\{0,1\}^{n}$ then $\operatorname{pad}_{n}(A)=A$. With $n$ understood we write $\operatorname{pad}(A)$ for $\operatorname{pad}_{n}(A)$. If $A=a_{n-1} a_{n-2} \cdots a_{1} a_{0} \in\{0,1\}^{n}$ then $A \ll 1=$ $a_{n-2} a_{n-3} \cdots a_{1} a_{0} 0$ is the $n$-bit string which is the left shift of $A$ by 1 bit while $A \gg 1=0 a_{n-1} a_{n-2} \ldots a_{2} a_{1}$ is the $n$-bit string which is the right shift of $A$ by one bit. In pseudocode we write "Partition $M$ into $M[1] \cdots M[m]$ " as shorthand for "Let $m=\|M\|_{n}$ and let $M[1], \ldots, M[m]$ be strings such that $M[1] \cdots M[m]=M$ and $|M[i]|=n$ for $1 \leq i<m$."

THE FIELD WITH $2^{n}$ POINTS. The field with $2^{n}$ points is denoted $\operatorname{GF}\left(2^{n}\right)$. We interchangeably think of a point $a$ in $\operatorname{GF}\left(2^{n}\right)$ in any of the following ways: (1) as an abstract point in the field; (2) as an $n$-bit string $a_{n-1} \ldots a_{1} a_{0} \in\{0,1\}^{n} ;(3)$ as a formal polynomial $a(\mathrm{x})=a_{n-1} \mathrm{x}^{n-1}+\cdots+a_{1} \mathrm{x}+a_{0}$ with binary coefficients; (4) as a nonnegative integer between 0 and $2^{n}-1$, where $a \in\{0,1\}^{n}$ corresponds to $\operatorname{str} 2$ num $(a)$. We write $a(\mathrm{x})$ instead of $a$ if we wish to emphasize that we are thinking of $a$ as a polynomial. To add two points in $\operatorname{GF}\left(2^{n}\right)$, take their bitwise xor. We denote this operation by $a \oplus b$. To multiply two points, fix some irreducible polynomial $p(\mathrm{x})$ having binary coefficients and degree $n$. To 
be concrete, choose the lexicographically first polynomial among the irreducible degree $n$ polynomials having a minimum number of coefficients. To multiply points $a, b \in \mathrm{GF}\left(2^{n}\right)$, which we denote $a \cdot b$, regard $a$ and $b$ as polynomials $a(\mathrm{x})=a_{n-1} \mathrm{x}^{n-1}+\cdots+a_{1} \mathrm{x}+a_{0}$ and $b(\mathrm{x})=b_{n-1} \mathrm{x}^{n-1}+\cdots+b_{1} \mathrm{x}+b_{0}$, form their product $c(\mathrm{x})$ where one adds and multiplies coefficients in $\mathrm{GF}(2)$, and take the remainder when dividing $c(\mathrm{x})$ by $p(\mathrm{x})$. Note that it is particularly easy to multiply a point $a \in\{0,1\}^{n}$ by $\mathrm{x}$. We illustrate the method for $n=128$, where $p(\mathrm{x})=\mathrm{x}^{128}+\mathrm{x}^{7}+\mathrm{x}^{2}+\mathrm{x}+1$. Then multiplying $a=a_{n-1} \cdots a_{1} a_{0}$ by $\mathrm{x}$ yields

$$
a \cdot \mathrm{x}= \begin{cases}a \ll 1 & \text { if } \operatorname{firstbit}(a)=0 \\ (a \ll 1) \oplus 0^{120} 10000111 & \text { if firstbit }(a)=1\end{cases}
$$

It is similarly easy to divide $a$ by $\mathrm{x}$ (meaning to multiply $a$ by the multiplicative inverse of $\mathrm{x}$ ). To illustrate, assume that $n=128$. Then

$$
a \cdot \mathbf{x}^{-1}= \begin{cases}a \gg 1 & \text { if lastbit }(a)=0 \\ (a \gg 1) \oplus 10^{120} 1000011 & \text { if lastbit }(a)=1\end{cases}
$$

If $L \in\{0,1\}^{n}$ and $i \geq-1$, we write $L(i)$ to mean $L \cdot \mathrm{x}^{i}$. To compute $L(-1), L(0), \ldots, L(\mu)$, where $\mu$ is small, set $L(0)=L$ and then, for $i \in[1 . . \mu]$, use Equation (1) to compute $L(i)=L(i-1) \cdot \mathrm{x}$ from $L(i-1)$; and use Equation $(2)$ to compute $L(-1)$ from $L$.

We point out that huge $=\mathrm{x}^{-1}$ will be an enormous number (when viewed as a number); in particular, huge starts with a 1 bit, so huge $>2^{n-1}$. In the security proof this fact is relevant, so there we use huge as a synonym for $\mathrm{x}^{-1}$ when this seems to add to clarity.

GRAY CODES. For any $\ell \geq 1$, a Gray code is an ordering $\gamma^{\ell}=\gamma_{0}^{\ell} \gamma_{1}^{\ell} \ldots \gamma_{2^{\ell-1}}^{\ell}$ of $\{0,1\}^{\ell}$ such that successive points differ (in the Hamming sense) by just one bit. For $n$ a fixed number, PMAC makes use of the "canonical" Gray code $\gamma=\gamma^{n}$ constructed by $\gamma^{1}=01$ while, for $\ell>0$,

$$
\gamma^{\ell+1}=\begin{array}{lllllllllll}
0 \gamma_{0}^{\ell} & 0 \gamma_{1}^{\ell} & \cdots & 0 \gamma_{2^{\ell}-2}^{\ell} & 0 \gamma_{2^{\ell}-1}^{\ell} & 1 \gamma_{2^{\ell}-1}^{\ell} & 1 \gamma_{2^{\ell}-2}^{\ell} & \cdots & 1 \gamma_{1}^{\ell} & 1 \gamma_{0}^{\ell} .
\end{array}
$$

It is easy to see that $\gamma$ is a Gray code. What is more, for $1 \leq i \leq 2^{n}-1$, $\gamma_{i}=\gamma_{i-1} \oplus\left(0^{n-1} 1 \ll \mathrm{ntz}(i)\right)$. This makes it easy to compute successive points. Note that $\gamma_{1}, \gamma_{2}, \ldots, \gamma_{2^{n}-1}$ are distinct, different from 0 , and $\gamma_{i} \leq 2 i$.

Let $L \in\{0,1\}^{n}$ and consider the problem of successively forming the strings $\gamma_{1} \cdot L, \gamma_{2} \cdot L, \gamma_{3} \cdot L, \ldots, \gamma_{m} \cdot L$. Of course $\gamma_{1} \cdot L=1 \cdot L=L$. Now, for $i \geq 2$, assume one has already produced $\gamma_{i-1} \cdot L$. Since $\gamma_{i}=\gamma_{i-1} \oplus\left(0^{n-1} 1 \ll \mathrm{ntz}(i)\right)$ we know that $\gamma_{i} \cdot L=\left(\gamma_{i-1} \oplus\left(0^{n-1} 1 \ll \mathrm{ntz}(i)\right)\right) \cdot L=\left(\gamma_{i-1} \cdot L\right) \oplus\left(0^{n-1} 1 \ll \mathrm{ntz}(i)\right) \cdot L=$ $\left(\gamma_{i-1} \cdot L\right) \oplus\left(L \cdot \mathrm{x}^{\mathrm{ntz}(i)}\right)=\left(\gamma_{i-1} \cdot L\right) \oplus L(\mathrm{ntz}(i))$. That is, the $i$ th word in the sequence $\gamma_{1} \cdot L, \gamma_{2} \cdot L, \gamma_{3} \cdot L, \ldots$ is obtained by xoring the previous word with $L(\operatorname{ntz}(i))$.

\section{Definition of PMAC}

PMAC depends on two parameters: a block cipher and a tag length. The block cipher is a function $E: \mathcal{K} \times\{0,1\}^{n} \rightarrow\{0,1\}^{n}$, for some number $n$, where each 


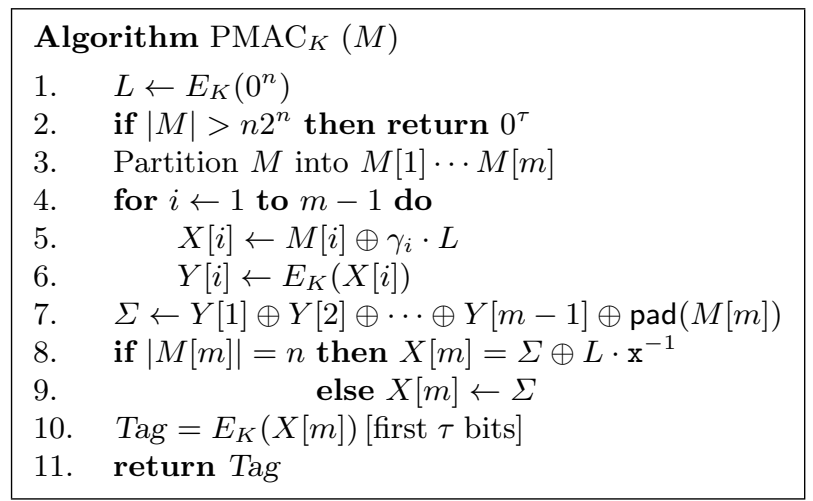

Fig. 1. Definition of PMAC. The message to MAC is $M$ and the key is $K$. The algorithm depends on a block cipher $E: \mathcal{K} \times\{0,1\}^{n} \rightarrow\{0,1\}^{n}$ and a number $\tau \in[1 . . n]$. Constants $\gamma_{1}, \gamma_{2}, \ldots$, the meaning of the multiplication operator, and the meaning of $\operatorname{pad}()$ are all defined in the text.

$E(K, \cdot)=E_{K}(\cdot)$ is a permutation on $\{0,1\}^{n}$. Here $\mathcal{K}$ is the set of possible keys and $n$ is the block length. The tag length is an integer $\tau \in[1 . . n]$. By trivial means the adversary will be able to forge a valid ciphertext with probability $2^{-\tau}$. With $E: \mathcal{K} \times\{0,1\}^{n} \rightarrow\{0,1\}^{n}$ and $\tau \in[1 . . n]$, we let PMAC $[E, \tau]$ denote PMAC using block cipher $E$ and tag length $\tau$. We simplify to PMAC- $E$ when $\tau$ is irrelevant. $\operatorname{PMAC}[E, \tau]$ is a function taking a key $K \in \mathcal{K}$ and a message $M \in\{0,1\}^{*}$ and returning a string in $\{0,1\}^{\tau}$. The function is defined in Figure 1 and illustrated in Figure 2. We comment that line 2 of Figure 1 is simply to ensure that PMAC is well-defined even for the highly unrealistic case that $|M|>n 2^{n}$ (by which time our security result becomes vacuous anyway). Alternatively, one may consider PMAC's message space to be strings of length at most $n 2^{n}$ rather than strings of arbitrary length.

\section{Comments}

As we shall soon prove, PMAC is more than a good MAC: it is good as a pseudorandom function (PRF) having variable-input-length and fixed-output-length. As long as the underlying block cipher $E$ is secure, no reasonable adversary will be able to distinguish $\operatorname{PMAC}_{K}(\cdot)$, for a random and hidden key $K$, from a random function $\rho$ from $\{0,1\}^{*}$ to $\{0,1\}^{\tau}$. It is a well-known observation, dating to the introduction of PRFs [15], that a good PRF is necessarily a good MAC.

Conceptually, the key is $(K, L)$. But instead of regarding this as the key (and possibly defining $K$ and $L$ from an underlying key), the value $L$ is defined from $K$ and then $K$ is still used as a key. Normally such "lazy key-derivation" would get one into trouble, in proofs if nothing else. For PMAC we prove that this form of lazy key-derivation works fine. 


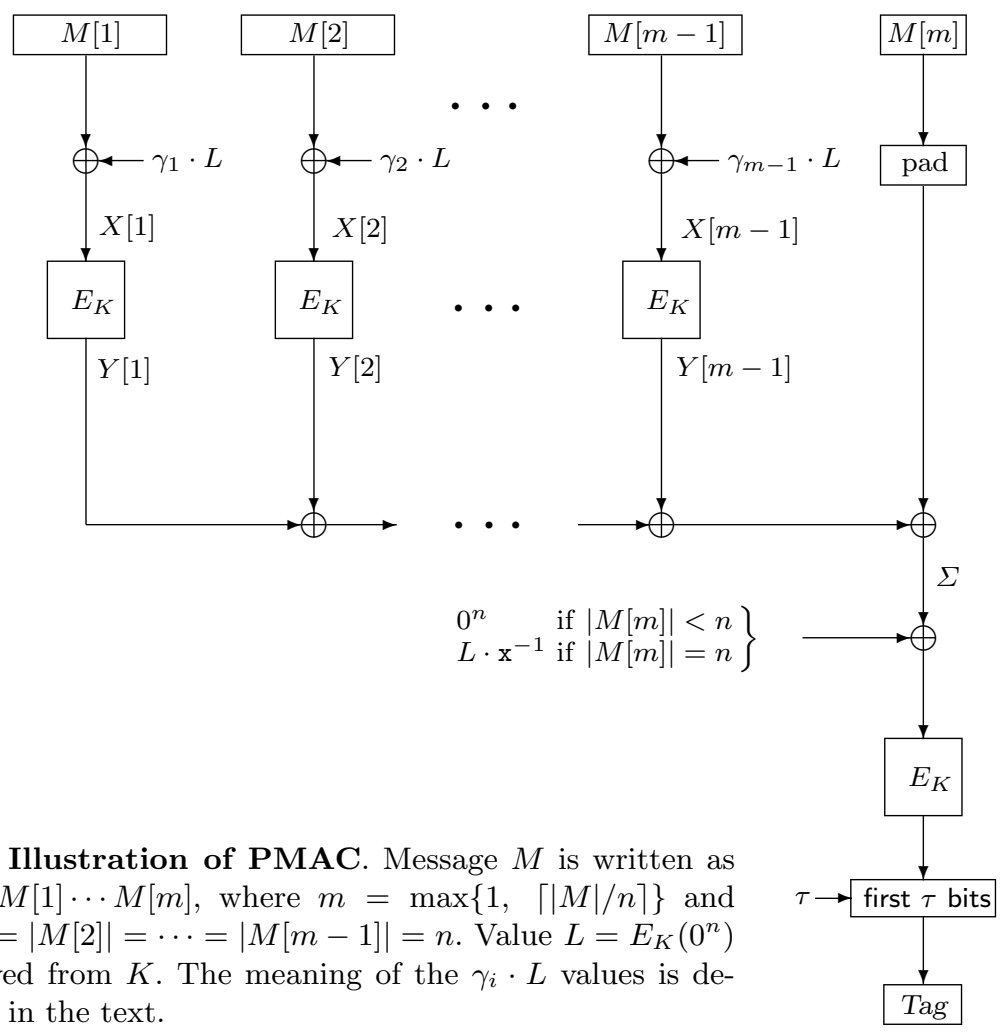

Fig. 2. Illustration of PMAC. Message $M$ is written as $M=M[1] \cdots M[m]$, where $m=\max \{1, \quad\lceil|M| / n\rceil\}$ and $|M[1]|=|M[2]|=\cdots=|M[m-1]|=n$. Value $L=E_{K}\left(0^{n}\right)$ is derived from $K$. The meaning of the $\gamma_{i} \cdot L$ values is described in the text.

Any string $M \in\{0,1\}^{*}$ can be MACed, and messages which are not a multiple of the block length are handled without the need for obligatory padding, which would increase the number of block-cipher calls.

MAC generation is "on line," meaning that one does not need to know the length of the message $M$ in advance. Instead, the message can be MACed as one goes along, continuing until there is an indication that the message is now complete. The work of Petrank and Rackoff brought out the importance of this property [21].

In contrast to a scheme based on $\bmod p$ arithmetic (for a prime $p$ ) or mod $2^{n}$ arithmetic, there is almost no endian-favoritism implicit in the definition of PMAC. (The exception is that the left shift used for forming $L(i+1)$ from $L(i)$ is more convenient under a big-endian convention, as is the right shift used for forming $L(-1)=L \cdot \mathrm{x}^{-1}$ from $L$.)

If $\tau=n$ (or one retains a constant amount of extra information) PMAC is incremental in the sense of [15] with respect to operations append $(M, x)=M \| x$, truncate $(M, \Delta)=M$ [first $|M|-\Delta$ bits], for $|M| \geq \Delta$, and $\operatorname{replace}(M, i, x)=$ $M$ [first $i-1$ bits] $\|x\| M$ [last $|M|-i-|x|+1$ bits], where $|M| \geq i+|x|-1)$. For each operation it is easy to see how to update the MAC of $M$ in time proportional to $|x|, \Delta$, or $|x|$, respectively. 
PMAC is parsimonious, as defined in [5]. Partition $M$ into $M[1] \cdots M[m]$ and assume $|M| \geq n$ and $\tau=n$. For $i \in[1 . . m]$ such that $|M[i]|=n$, there is a simple algorithm to recover $M[i]$ from $K, M^{\prime}=M[1] \cdots M[i-1] M[i+1] \cdots M[m]$, and $\mathrm{Tag}=\mathrm{PMAC}_{K}(M)$. As shown in [5], a parsimonious PRF can be combined with a parsimonious encryption scheme (eg., CTR mode) to yield a lengthpreserving pseudorandom permutation (a "variable-input-length block cipher") that acts on messages of any number of bits greater than or equal to $n$.

\section{Theorems}

SECURITy DEFinitions. We first recall the needed definitions. A block cipher is a function $E: \mathcal{K} \times\{0,1\}^{n} \rightarrow\{0,1\}^{n}$ where $\mathcal{K}$ is a finite set and each $E_{K}(\cdot)=$ $E(K, \cdot)$ is a permutation on $\{0,1\}^{n}$. Let $\operatorname{Perm}(n)$ denote the set of all permutations on $\{0,1\}^{n}$. This set can be regarded as a block cipher by imagining that each permutation is named by a unique element of $\mathcal{K}$. Let $A$ be an adversary (a probabilistic algorithm) with access to an oracle, and suppose that $A$ always outputs a bit. Define

$$
\mathbf{A d v}_{E}^{\operatorname{prp}}(A)=\operatorname{Pr}\left[K \stackrel{R}{\leftarrow} \mathcal{K}: A^{E_{K}(\cdot)}=1\right]-\operatorname{Pr}\left[\pi \stackrel{R}{\leftarrow} \operatorname{Perm}(n): A^{\pi(\cdot)}=1\right]
$$

The above is the probability that adversary $A$ outputs 1 when given an oracle for $E_{K}(\cdot)$, minus the probability that $A$ outputs 1 when given an oracle for $\pi(\cdot)$, where $K$ is selected at random from $\mathcal{K}$ and $\pi$ is selected at random from $\operatorname{Perm}(n)$. Similarly, a function family from $n$-bits to $n$-bits is a map $F: \mathcal{K} \times\{0,1\}^{n} \rightarrow$ $\{0,1\}^{n}$ where $\mathcal{K}$ is a finite set. We write $F_{K}(\cdot)$ for $F(K, \cdot)$. Let $\operatorname{Rand}(n)$ denote the set of all functions from $\{0,1\}^{n}$ to $\{0,1\}^{n}$. This set can be regarded as a function family as above. Define

$$
\mathbf{A d v}_{F}^{\operatorname{prf}}(A)=\operatorname{Pr}\left[K \stackrel{R}{\leftarrow} \mathcal{K}: A^{F_{K}(\cdot)}=1\right]-\operatorname{Pr}\left[\rho \stackrel{R}{\leftarrow} \operatorname{Rand}(n): A^{\rho(\cdot)}=1\right]
$$

Finally, a function family from $\{0,1\}^{*}$ to $\{0,1\}^{\tau}$ is a map $f: \mathcal{K} \times\{0,1\}^{*} \rightarrow\{0,1\}^{\tau}$ where $\mathcal{K}$ is a set with an associated distribution. We write $f_{K}(\cdot)$ for $f(K, \cdot)$. Let $\operatorname{Rand}(*, \tau)$ denote the set of all functions from $\{0,1\}^{*}$ to $\{0,1\}^{\tau}$. This set is given a probability measure by asserting that a random element $\rho$ of $\operatorname{Rand}(*, \tau)$ associates to each string $x \in\{0,1\}^{*}$ a random string $\rho(x) \in\{0,1\}^{\tau}$. Define

$$
\mathbf{A d v}_{f}^{\text {prf }}(A)=\operatorname{Pr}\left[K \stackrel{R}{\leftarrow} \mathcal{K}: A^{f_{K}(\cdot)}=1\right]-\operatorname{Pr}\left[g \stackrel{R}{\leftarrow} \operatorname{Rand}(*, \tau): A^{g(\cdot)}=1\right]
$$

MAIN RESULT. We now give an information-theoretic bound on the security of our construction.

Theorem 1. [Security of PMAC] Fix $n, \tau \geq 1$. Let $A$ be an adversary with an oracle. Suppose that $A$ asks its oracle $q$ queries, these having aggregate length of $\sigma$ blocks. Let $\bar{\sigma}=\sigma+1$. Then

$$
\operatorname{Adv}_{\operatorname{PMAC}[\operatorname{Perm}(n), \tau]}^{\operatorname{prf}}(A) \leq \frac{\bar{\sigma}^{2}}{2^{n-1}}
$$




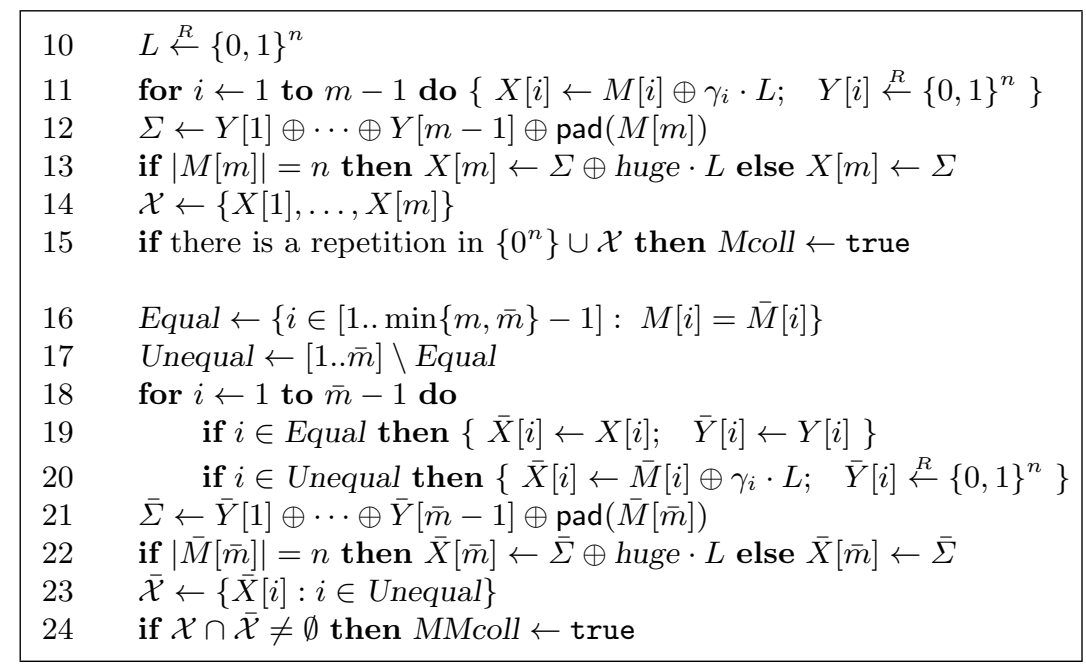

Fig. 3. Defining the collision probabilities. Functions $\operatorname{Mcoll}_{n}(\cdot)$ and $\operatorname{MMcoll}_{n}(\cdot, \cdot)$ are defined using this game. In lines 14 and $23, \mathcal{X}$ and $\overline{\mathcal{X}}$ are understood to be multisets. The union in line 15 is a multiset union. Recall that huge is a synonym for $\mathrm{x}^{-1}$.

In the theorem statement and from now on, the aggregate length of messages $M_{1}, \ldots, M_{q}$ asked by $A$ is the number $\sigma=\sum_{r=1}^{q}\left\|M_{r}\right\|_{n}$.

From the theorem above it is standard to pass to a complexity-theoretic analog. Fix parameters $n, \tau \geq 1$ and block cipher $E: \mathcal{K} \times\{0,1\}^{n} \rightarrow\{0,1\}^{n}$. Let $A$ be an adversary with an oracle and suppose that $A$ asks queries having aggregate length of $\sigma$ blocks. Let $\bar{\sigma}=\sigma+1$. Then there is an adversary $B$ for attacking $E$ that achieves advantage $\mathbf{A d v}_{E}^{\text {prp }}(B) \geq \mathbf{A} \mathbf{d v}_{\operatorname{PMAC}[E, \tau]}^{\operatorname{prf}}(A)-\bar{\sigma}^{2} / 2^{n-1}$. Adversary $B$ asks at most $\bar{\sigma}$ oracle queries and has a running time equal to $A$ 's running time plus the time to compute $E$ on $\bar{\sigma}$ points, plus additional time of $c n \bar{\sigma}$ for a constant $c$ that depends only on details of the model of computation.

It is a standard result that being secure in the sense of a PRF implies an inability to forge with good probability. See $[4,15]$.

Structure of the Proof. The proof combines two lemmas. The first, the structure lemma, measures the pseudorandomness of PMAC using two functions: the M-collision probability, denoted $\operatorname{Mcoll}_{n}(\cdot)$, and the MM-collision probability, denoted $\operatorname{MMcoll}_{n}(\cdot, \cdot)$. The second lemma, the collision-bounding lemma, upperbounds $\operatorname{Mcoll}_{n}(m)$ and $\operatorname{MMcoll}_{n}(m, \bar{m})$.

We begin by defining $\operatorname{Mcoll}_{n}(\cdot)$ and $\operatorname{MMcoll}_{n}(\cdot, \cdot)$. Fix $n$ and choose $M$ and $\bar{M}$, partitioning them into $M[1] \cdots M[m]$ and $\bar{M}[1] \cdots \bar{M}[\bar{m}]$. Consider the experiment of Figure 3. When $M$ is a string, let $\operatorname{Mcoll}_{n}(M)$ denote the probability that Mcoll gets set to true in line 15 when the program of Figure 3 is run on $M$. When $m$ is a number, $\operatorname{Mcoll}_{n}(m)$ is the maximum value of $\operatorname{Mcoll}_{n}(M)$ over all strings $M$ such that $\|M\|_{n}=m$. Similarly, when $M$ and $\bar{M}$ are strings, let $\operatorname{MMcoll}_{n}(M, \bar{M})$ denote the probability that MMcoll gets set to true when the 
program of Figure 3 is run on strings $M, \bar{M}$. When $m$ and $\bar{m}$ are numbers, let $\operatorname{MMcoll}_{n}(m, \bar{m})$ denote the maximum value of $\operatorname{MMcoll}_{n}(M, \bar{M})$ over all strings $M, \bar{M}$ such that $\|M\|_{n}=m$ and $\|\bar{M}\|_{n}=\bar{m}$. We can now state the structure lemma.

Lemma 1. [Structure lemma] Fix $n, \tau \geq 1$. Let $A$ be an adversary that asks $q$ queries, these having an aggregate length of $\sigma$ blocks. Then

$$
\begin{aligned}
& \operatorname{Adv}_{\mathrm{PMAC}[\operatorname{Perm}(n), \tau]}^{\mathrm{prf}}(A) \leq \\
& \underset{\substack{m_{1}, \ldots, m_{q} \\
\sigma=\sum_{i} m_{i} \\
m_{i} \geq 1}}{\operatorname{Pax}}\left\{\sum_{1 \leq r \leq q} \operatorname{Mcoll}_{n}\left(m_{r}\right)+\sum_{1 \leq r<s \leq q} \operatorname{MMcoll}_{n}\left(m_{r}, m_{s}\right)\right\}+\frac{(\sigma+1)^{2}}{2^{n+1}}
\end{aligned}
$$

The proof of this lemma is found in [10].

ExPLANATION. Informally, $\operatorname{Mcoll}_{n}(m)$ measures the probability of running into trouble when the adversary asks a single question $M$ having block length $m$. Trouble means a collision among the values $X[0], X[1], \ldots, X[m]$, where $X[0]=$ $0^{n}$ and each $X[i]$ is the block-cipher input associated to message block $i$. Informally, $\operatorname{MMcoll}_{n}(m, \bar{m})$ measures the probability of running into trouble across two messages, $M$ and $\bar{M}$, having lengths $m$ and $\bar{m}$. This time trouble means a "non-trivial" collision. That is, consider the $m+\bar{m}+1$ points at which the block cipher is applied in processing $M$ and $\bar{M}$. There are $m$ points $X[1], \ldots, X[m]$, another $\bar{m}$ points $\bar{X}[1], \ldots, \bar{X}[\bar{m}]$, and then there is the point $0^{n}$ (the block cipher was applied at this point to define $L)$. Some pairs of these $m+\bar{m}+1$ points could coincide for a "trivial" reason: namely, we know that $X[i]=\bar{X}[i]$ if $i<m$ and $i<\bar{m}$ and $M[i]=\bar{M}[i]$. We say that there is a nontrivial collision between $M$ and $\bar{M}$ if some other $X[i]$ and $\bar{X}[j]$ happened to coincide. Note that M-collisions include collisions with $0^{n}$, while MM-collisions do not. Also, MMcollisions do not include collisions within a single message (or collisions with $0^{n}$ ) because both of these possibilities are taken care of by way of M-collisions.

The structure lemma provides a simple recipe for measuring the maximum advantage of any adversary that attacks the pseudorandomness of PMAC: bound the collision probabilities $\operatorname{Mcoll}_{n}(\cdot)$ and $\operatorname{MMcoll}_{n}(\cdot, \cdot)$ and then use the formula. The lemma simplifies the analysis of PMAC in two ways. First, it allows one to excise adaptivity as a concern. Dealing with adaptivity is a major complicating factor in proofs of this type. Second, it allows one to concentrate on what happens to single messages and to a fixed pair of messages. It is easier to think about what happens with one or two messages than what is happening with all $q$ of them.

Bounding THE COLLISION PROBABILITIEs. The following lemma indicates that the two types of collisions we have defined rarely occur. The proof shall be given shortly. 
Lemma 2. [Collision-bounding lemma] Let $\operatorname{Mcoll}_{n}(\cdot)$ and $\operatorname{MMcoll}_{n}(\cdot, \cdot)$ denote the M-collision probability and the MM-collision probability. Then

$$
\operatorname{Mcoll}_{n}(m) \leq\left(\begin{array}{c}
m+1 \\
2
\end{array}\right) \cdot \frac{1}{2^{n}} \quad \text { and } \quad \operatorname{MMcoll}_{n}(m, \bar{m}) \leq \frac{m \bar{m}}{2^{n}}
$$

Concluding The Theorem. Pseudorandomness of PMAC, Theorem 1, follows by combining Lemmas 1 and 2. Namely,

$$
\begin{aligned}
& \mathbf{A d} \mathbf{v}_{\operatorname{PMAC}[\operatorname{Perm}(n), \tau]}^{\operatorname{prf}} \\
& \leq \max _{\substack{m_{1}, \ldots, m_{q} \\
\sigma=\sum_{i} \\
m_{i} \geq 1}}\left\{\sum_{1 \leq r \leq q} \operatorname{Mcoll}_{n}\left(m_{r}\right)+\sum_{1 \leq r<s \leq q} \operatorname{MMcoll}_{n}\left(m_{r}, m_{s}\right)\right\}+\frac{(\sigma+1)^{2}}{2^{n+1}} \\
& \leq \max _{\substack{m_{1}, \ldots, m_{q} \\
\sigma=m_{i} \\
m_{i} \geq 1}}\left\{\sum_{1 \leq r \leq q} \operatorname{Mcoll}_{n}\left(m_{r}\right)\right\} \\
& +\max _{\substack{m_{1}, \ldots, m_{q} \\
\sigma=m_{i} \\
m_{i} \geq 0}}\left\{\sum_{\substack{1 \leq r<s \leq q \\
1 \leq m^{2}}} \operatorname{MMcoll}_{n}\left(m_{r}, m_{s}\right)\right\}+\frac{(\sigma+1)^{2}}{2^{n+1}} \\
& \leq \max _{\substack{m_{1}, \ldots, m_{q} \\
\sigma=\sum_{i} m_{i} \\
m_{i} \geq 0}}\left\{\sum_{1 \leq r \leq q}\left(\begin{array}{c}
m_{r}+1 \\
2
\end{array}\right) \cdot \frac{1}{2^{n}}\right\} \\
& +\max _{\substack{m_{1}, \ldots, m_{q} \\
\sigma=m_{i} \\
m_{i} \geq 0}}\left\{\sum_{\substack{1 \leq r<s \leq q \\
m_{i}}} \frac{m_{r} m_{s}}{2^{n}}\right\}+\frac{(\sigma+1)^{2}}{2^{n+1}} \\
& \leq \frac{(\sigma+1)^{2}}{2^{n}}+\frac{\left(\sigma^{2} / 2\right)}{2^{n}}+\frac{(\sigma+1)^{2}}{2^{n+1}} \\
& \leq \frac{2(\sigma+1)^{2}}{2^{n}}
\end{aligned}
$$

where (3) follows because the first sum is maximized with a single message of length $\sigma$, while the second sum is maximized by $q$ messages of length $\sigma / q$. (These claims can be justified using the method of Lagrange multipliers.) This completes the proof of Theorem 1 .

Proof of Lemma 2. We now bound $\operatorname{Mcoll}_{n}(m)$ and $\operatorname{MMcoll}_{n}(m, \bar{m})$. To begin, let Unequal $^{\prime}=$ Unequal $\backslash\{\bar{X}[\bar{m}]\}$ (multiset difference: remove one copy of $\bar{X}[\bar{m}]$ ) and define

$$
\begin{gathered}
D_{1}=\left\{0^{n}\right\} \quad D_{2}=\{X[1], \ldots, X[m-1]\} \quad D_{3}=\{X[m]\} \\
D_{4}=\left\{\bar{X}[j]: j \in \text { Unequal }^{\prime}\right\} \quad D_{5}=\{\bar{X}[\bar{m}]\}
\end{gathered}
$$

We first show that for any two points $X[i]$ and $X[j]$ in the multiset $D_{1} \cup D_{2} \cup D_{3}$, where $i<j$ and $X[0]=0^{n}$, the probability that these two points collide is at 
most $2^{-n}$. The inequality follows because there are $m+1$ points in $D_{1} \cup D_{2} \cup D_{3}$. Afterwards, we show that for any point $X[i]$ in $D_{2} \cup D_{3}$ and any point in $\bar{X}[j]$ in $D_{4} \cup D_{5}$, the probability that they collide is at most $2^{-n}$. The inequality $\operatorname{MMcoll}_{n}(m, \bar{m}) \leq \frac{m \bar{m}}{2^{n}}$ follows because $\left|D_{2} \cup D_{3}\right| \cdot\left|D_{4} \cup D_{5}\right| \leq m \bar{m}$.

To show $\operatorname{Mcoll}_{n}(m) \leq\left(\begin{array}{c}m+1 \\ 2\end{array}\right) \cdot \frac{1}{2^{n}}$ consider the following four cases:

$\operatorname{CASE}\left(D_{1}, D_{2}\right): \quad \operatorname{Pr}\left[0^{n}=X[i]\right]=\operatorname{Pr}\left[M[i] \oplus \gamma_{i} \cdot L=0^{n}\right]=\operatorname{Pr}\left[L=\gamma_{i}^{-1} \cdot M[i]\right]=$ $2^{-n}$. We have used that $\gamma_{i}$ is nonzero and we are working in a field. (We will continue to use this without mention.)

CASE $\left(D_{1}, D_{3}\right):$ If $|M[m]|<n$ and $m \geq 2$ then $\Sigma$ is a random $n$-bit string and so $\operatorname{Pr}\left[0^{n}=X[m]\right]=\operatorname{Pr}\left[0^{n}=\Sigma\right]=\operatorname{Pr}\left[0^{n}=Y[1] \oplus \cdots Y[m-1] \oplus \operatorname{pad}(M[m])\right]=$ $2^{-n}$. If $|M[m]|=n$ and $m \geq 2$ then $\Sigma$ is a random $n$-bit string that is independent of $L$ and so $\operatorname{Pr}\left[0^{n}=X[m]\right]=\operatorname{Pr}\left[0^{n}=\Sigma \oplus\right.$ huge $\left.\cdot L\right]=2^{-n}$. If $|M[m]|<n$ and $m=1$ then $\operatorname{Pr}\left[0^{n}=X[1]\right]=\operatorname{Pr}\left[0^{n}=\operatorname{pad}(M[m])\right]=0$. If $|M[m]|=n$ and $m=1$ then $\operatorname{Pr}\left[0^{n}=X[1]\right]=\operatorname{Pr}\left[0^{n}=\operatorname{pad}(M[m]) \oplus\right.$ huge $\left.\cdot L\right]=2^{-n}$.

$\operatorname{CAse}\left(D_{2}, D_{2}\right):$ For $i, j \in[1 . . m-1], i<j, \operatorname{Pr}[X[i]=X[j]]=\operatorname{Pr}\left[M[i] \oplus \gamma_{i} \cdot L=\right.$ $\left.M[j] \oplus \gamma_{j} \cdot L\right]=\operatorname{Pr}\left[M[i] \oplus M[j]=\left(\gamma_{i} \oplus \gamma_{j}\right) \cdot L\right]=2^{-n}$ because $\gamma_{i} \neq \gamma_{j}$ for $i \neq j$. (Here one assumes that $j<2^{n}$ because the lemma gives a non-result anyway if $j$ were larger.)

CAse $\left(D_{2}, D_{3}\right): \quad$ Assume that $m \geq 2$, for otherwise there is nothing to show. Suppose first that $|M[m]|<n$. Then $\operatorname{Pr}[X[i]=X[m]]=\operatorname{Pr}\left[M[i] \oplus \gamma_{i} \cdot L=\Sigma\right]$. The value $\Sigma$ is uniformly random and independent of $L$, so this probability is $2^{-n}$. Suppose next that $|M[m]|=n$. Then $\operatorname{Pr}[X[i]=X[m]]=\operatorname{Pr}\left[M[i] \oplus \gamma_{i} \cdot L=\right.$ $\Sigma \oplus$ huge $\cdot L]=\operatorname{Pr}\left[M[i] \oplus \Sigma=\left(\gamma_{i} \oplus\right.\right.$ huge $\left.) \cdot L\right]$. This value is $2^{-n}$ since $\gamma_{i} \neq$ huge. Here we are assuming that $i<2^{n-1}$, which is without loss of generality since a larger value of $i$, and therefore $m$, would give a non-result in the theorem statement.

Moving on, to show that $\operatorname{MMcoll}_{n}(m, \bar{m}) \leq \frac{m \bar{m}}{2^{n}}$ we verify the following four cases:

CAse $\left(D_{2}, D_{4}\right):$ Let $i \in[1 . . m-1]$ and $j \in$ Unequal $^{\prime}$ and consider $\operatorname{Pr}[X[i]=$ $\bar{X}[j]]=\operatorname{Pr}\left[M[i] \oplus \gamma_{i} \cdot L=\bar{M}[j] \oplus \gamma_{j} \cdot L\right]=\operatorname{Pr}\left[M[i] \oplus \bar{M}[j]=\left(\gamma_{i} \oplus \gamma_{j}\right) \cdot L\right.$. If $i \neq j$ then $\gamma_{i} \neq \gamma_{j}$ and this probability is $2^{-n}$. If $i=j$ then the probability is 0 since, necessarily, $M[i] \neq \bar{M}[j]$.

CASE $\left(D_{2}, D_{5}\right): \quad$ Suppose that $|\bar{M}[\bar{m}]|<n$. Then $\operatorname{Pr}[X[i]=\bar{X}[\bar{m}]]=\operatorname{Pr}[M[i] \oplus$ $\left.\gamma_{i} \cdot L=\bar{\Sigma}\right]=2^{-n}$ because $\bar{\Sigma}$ is independent of $L$. Suppose that $|\bar{M}[\bar{m}]|=n$. Then $\operatorname{Pr}[X[i]=\bar{X}[\bar{m}]]=\operatorname{Pr}\left[M[i] \oplus \gamma_{i} \cdot L=\bar{\Sigma} \oplus\right.$ huge $\left.\cdot L\right]=\operatorname{Pr}[M[i] \oplus \bar{\Sigma}=$ $\left(\gamma_{i} \oplus\right.$ huge $\left.) \cdot L\right]=2^{-n}$ because $\bar{\Sigma}$ is independent of $L$ and $\gamma_{i} \neq$ huge.

CAse $\left(D_{3}, D_{4}\right):$ Suppose that $|M[m]|<n$. Then $\operatorname{Pr}[X[m]=\bar{X}[j]]=\operatorname{Pr}[\Sigma=$ $\left.\bar{M}[j] \oplus \gamma_{j} \cdot L\right]=2^{-n}$ because $\Sigma$ is independent of $L$. Suppose that $|M[m]|=n$. Then $\operatorname{Pr}[X[m]=\bar{X}[j]]=\operatorname{Pr}\left[\Sigma \oplus\right.$ huge $\left.\cdot L=\bar{M}[j] \oplus \gamma_{j} \cdot L\right]=\operatorname{Pr}[\Sigma \oplus \bar{M}[j]=$ $\left(\gamma_{j} \oplus\right.$ huge $\left.) \cdot L\right]=2^{-n}$ because $\gamma_{j} \neq$ huge. 


\begin{tabular}{|l|r|r|r|}
\hline Algorithm & $16 \mathrm{~B}$ & $128 \mathrm{~B}$ & $2 \mathrm{~KB}$ \\
\hline \hline PMAC-AES128 & 22.1 & 18.7 & 18.4 \\
\hline CBCMAC-AES128 & 18.9 & 17.4 & 17.1 \\
\hline
\end{tabular}

Fig. 4. Performance results. Numbers are in cycles per byte (cpb) on a Pentium 3, for three message lengths, the code written in assembly.

CASE $\left(D_{3}, D_{5}\right)$ : Suppose that $|M[m]|<n$ and $|\bar{M}[\bar{m}]|<n$. If $m>\bar{m}$ then $\operatorname{Pr}[X[m]=\bar{X}[\bar{m}]]=\operatorname{Pr}[\Sigma=\bar{\Sigma}]=2^{-n}$ because of the contribution of $Y[m-1]$ in $\Sigma$ - a random variable that is not used in the definition of $\bar{\Sigma}$. If $m<\bar{m}$ then $\operatorname{Pr}[X[m]=\bar{X}[\bar{m}]]=\operatorname{Pr}[\Sigma=\bar{\Sigma}]=2^{-n}$ because of the contribution of $\bar{Y}[\bar{m}-1]$ in $\bar{\Sigma}$ - a random variable that is not used in the definition of $\Sigma$. If $m=\bar{m}$ and there is an $i<m$ such that $M[i] \neq \bar{M}[i]$ then $\operatorname{Pr}[X[m]=\bar{X}[\bar{m}]]=\operatorname{Pr}[\Sigma=\bar{\Sigma}]=2^{-n}$ because of the contribution of $\bar{Y}[i]$ in $\bar{\Sigma}$ - a random variable that is not used in the definition of $\Sigma$. If $m=\bar{m}$ and for every $i<m$ we have that $M[i]=\bar{M}[i]$, then, necessarily, $M[m] \neq \bar{M}[m]$. In this case $\operatorname{Pr}[\Sigma=\bar{\Sigma}]=0$, as the two checksums differ by the nonzero value $\operatorname{pad}(M[m]) \oplus \operatorname{pad}(\bar{M}[m])$.

Suppose that $|M[m]|=n$ and $|\bar{M}[\bar{m}]|=n$. Then $X[m]$ and $\bar{X}[m]$ are offset by the same amount, huge $L$, so this offset is irrelevant in computing $\operatorname{Pr}[X[m]=$ $\bar{X}[\bar{m}]$. Proceed as above.

Suppose that $|M[m]|<n$ and $|\bar{M}[\bar{m}]|=n$. Then $\operatorname{Pr}[X[m]=\bar{X}[m]]=\operatorname{Pr}[\Sigma=$ $\bar{\Sigma} \oplus$ huge $\cdot L]=2^{-n}$ since $\Sigma$ and $\bar{\Sigma}$ are independent of $L$. Similarly, if $|M[m]|=n$ and $|\bar{M}[\bar{m}]|<n$, then $\operatorname{Pr}[X[m]=\bar{X}[m]]=2^{-n}$. This completes the proof.

\section{Performance}

A colleague, Ted Krovetz, implemented PMAC-AES128 and compared its performance in an entirely sequential setting to that of CBCMAC-AES128. The latter refers to the "basic" CBC MAC; nothing is done to take care of length-variability or the possibility of strings which are not a multiple of the block length. The code was written in modestly-optimized assembly under Windows $2000 \mathrm{sp} 1$ and Visual C++ $6.0 \mathrm{sp} 4$. All data fit into L1 cache. Disregarding the one-block message in Figure 4 we see that, in a serial environment, PMAC-AES128 was about $8 \%$ slower than CBCMAC-AES128. A more aggressively optimized implementation of CBCMAC-AES128, due to Helger Lipmaa, achieves $15.5 \mathrm{cpb}$ for 1 KByte message lengths [20]. Adding the same $8 \%$, we expect that this code could be modified to compute PMAC at a rate of about $16.7 \mathrm{cpb}$. In general, differences in implementation quality would seem to be a more significant in determining implementation speed than the algorithmic difference between PMAC and the CBC MAC.

Though some or all of the $L(i)$-values are likely to be pre-computed, calculating all of these values "on the fly" is not expensive. Starting with $0^{n}$ we form successive offsets by xoring the previous offset with $L, 2 \cdot L, L, 4 \cdot L, L, 2 \cdot L, L$, $8 \cdot L$, and so forth, making the expected number of $a \cdot \mathrm{x}$-operations to compute 
an offset at most $\sum_{i=1}^{\infty} i / 2^{i+1}=1$. For $n=128$, each $a \cdot \mathrm{x}$ instruction requires a 128-bit shift and a conditional 32-bit xor.

\section{Acknowledgments}

We would like to thank several people for helpful comments on drafts of this manuscript: Michael Amling, Mihir Bellare, Johan Håstad, Ted Krovetz, David McGrew, David Wagner, and the Eurocrypt program committee. Special thanks to Ted, who wrote reference code, prepared test vectors, and collected performance data. Virgil Gligor described [14] to Rogaway at Crypto '00 and that event, along with the announcement of a NIST modes-of-operation workshop in October 2000, inspired the current work.

This paper was written while Rogaway was on leave from UC Davis, visiting the Department of Computer Science, Faculty of Science, Chiang Mai University.

\section{References}

1. M. Bellare, R. Canetti, and H. Krawczyk. Keying hash functions for message authentication. Advances in Cryptology - CRYPTO '96. Lecture Notes in Computer Science, vol. 1109, Springer-Verlag, pp. 1-15, 1996. Available at URL www-cse.ucsd.edu/users/mihir

2. M. Bellare, S. Goldwasser, and O. Goldreich. Incremental cryptography and applications to virus protection. Proceedings of the 27th Annual ACM Symposium on the Theory of Computing (STOC '95). ACM Press, pp. 45-56, 1995. Available at URL www.cs.ucdavis.edu/ rogaway

3. M. Bellare, R. Guérin and P. Rogaway. "XOR MACs: New methods for message authentication using finite pseudorandom functions." Advances in Cryptology - CRYPTO '95. Lecture Notes in Computer Science, vol. 963, Springer-Verlag, pp. 15-28, 1995. Available at URL www.cs.ucdavis.edu/ rogaway

4. M. Bellare, J. Kilian, and P. Rogaway. The security of the cipher block chaining message authentication code. Journal of Computer and System Sciences, vol. 61, no. 3, Dec 2000. (Full version of paper from Advances in Cryptology CRYPTO '94. Lecture Notes in Computer Science, vol. 839, pp. 340-358, 1994.) Available at URL www.cs.ucdavis.edu/ rogaway

5. M. Bellare and P. Rogaway. Encode-then-encipher encryption: How to exploit nonces or redundancy in plaintexts for efficient encryption. Advances in Cryptology - ASIACRYPT '00. Lecture Notes in Computer Science, vol. 1976, SpringerVerlag, 2000. Available at URL www.cs.ucdavis.edu/ rogaway

6. A. Berendschot, B. den Boer, J.P. Boly, A. Bosselaers, J. Brandt, D. Chaum, I. Damgård, M. Dichte, W. Fumy, M. van der Ham, C.J.A. Jansen, P. Landrock, B. Preneel, G. Roelofsen, P. De Rooij, and J. Vandewalle. Integrity primitives for secure information systems, Final report of RACE integrity primitives evaluation (RIPE-RACE 1040). Lecture Notes in Computer Science, vol. 1007, Springer-Verlag, 1995.

7. D. Bernstein. Floating-point arithmetic and message authentication. Unpublished manuscript. Available at URL http://cr.yp.to/papers.html\#hash127

8. D. BERnsteIn. How to stretch random functions: the security of protected counter sums. Journal of Cryptology, vol. 12, no. 3, pp. 185-192 (1999). Available at URL cr.yp.to/djb.html 
9. J. Black, S. Halevi, H. Krawczyk, T. Krovetz, and P. Rogaway. UMaC: Fast and secure message authentication. Advances in Cryptology - CRYPTO '99. Lecture Notes in Computer Science, Springer-Verlag, 1999. Available at URL www.cs.ucdavis.edu/ rogaway

10. J. Black and P. Rogaway. A block-cipher mode of operation for parallelizable message authentication. Full version of this paper. Available at URL www.cs.ucdavis.edu/ rogaway

11. J. Black and P. Rogaway. CBC MACs for arbitrary-length messages: The three-key constructions. Full version of paper from Advances in Cryptology CRYPTO '00. Lecture Notes in Computer Science, vol. 1880, pp. 197-215, 2000. Available at URL www.cs.ucdavis.edu/ rogaway

12. G. BRASSARD. On computationally secure authentication tags requiring short secret shared keys. Advances in Cryptology - CRYPTO '82. Plenum Press, pp. 7986, 1983.

13. L. Carter and M. Wegman. Universal hash functions. J. of Computer and System Sciences. vol. 18, pp. 143-154, 1979.

14. V. Gligor and P. Donescu. Fast encryption and authentication: XCBC encryption and XECB authentication modes. Fast Software Encryption, Lecture Notes in Computer Science, Springer-Verlag, April 2001. Available at URL www.eng.umd.edu/ gligor

15. O. Goldreich, S. Goldwasser, and S. Micali. How to construct random functions. Journal of the ACM, vol. 33, no. 4, pp. 210-217, 1986.

16. S. Halevi and H. KraWczyK. MMH: Software message authentication in the Gbit/second rates. Fast Software Encryption (FSE 4), Lecture Notes in Computer Science, vol. 1267, Springer-Verlag, pp. 172-189, 1997. Available at URL www.research.ibm.com/people/s/shaih

17. Iso/IEC 9797. Information technology - Security techniques - Data integrity mechanism using a cryptographic check function employing a block cipher algorithm. International Organization for Standards (ISO), Geneva, Switzerland, 1994 (second edition).

18. C. Jutla. Encryption modes with almost free message integrity. Advances in Cryptology - EUROCRYPT 2001. Lecture Notes in Computer Science, vol. 2045, B. Pfitzmann, ed., Springer-Verlag, 2001.

19. H. KRAWCZYK. LFSR-based hashing and authentication. Advances in Cryptology - CRYPTO '94. Lecture Notes in Computer Science, vol. 839, Springer-Verlag, pp 129-139, 1994.

20. H. LipmaA. Personal communication, July 2001. Further information available at www.tcs.hut.fi/ helger

21. E. Petrank and C. RACkoff. CBC MAC for real-time data sources. Journal of Cryptology, vol. 13, no. 3, pp. 315-338, Nov 2000. Available at URL www.cs.technion.ac.il/ erez/publications.html. Earlier version as 1997/010 in the Cryptology ePrint archive, eprint.iacr.org

22. B. Preneel. Cryptographic primitives for information authentication - State of the art. State of the Art in Applied Cryptography, COSIC '97, LNCS 1528, B. Preneel and V. Rijmen, eds., Springer-Verlag, pp. 49-104, 1998.

23. M. WEgman and L. CARTER. New hash functions and their use in authentication and set equality. J. of Comp. and System Sciences. vol. 22, pp. 265-279, 1981. 